\title{
Hysteretic structural changes within five-layered modulated 10M martensites of $\mathrm{Ni}-\mathrm{Mn}$-Ga(-Fe)
}

\author{
P. Veřtát ${ }^{1,2}$, L. Straka ${ }^{1}$, H. Seiner ${ }^{3}$, A. Sozinov ${ }^{4}$, M. Klicpera ${ }^{5}$, O. Fabelo ${ }^{6}$, O. Heczko ${ }^{1}$
}

${ }^{1}$ Institute of Physics of the Czech Academy of Sciences, Na Slovance 1999/2, 18221 Prague 8, Czech Republic, ${ }^{2}$ Faculty of Nuclear

Sciences and Physical Engineering, Czech Technical University in Prague, Trojanova 13, 12000 Prague 2, Czech Republic, ${ }^{3}$ Institute of Thermomechanics of the Czech Academy of Sciences, Dolejškova 1402/8, 18200 Prague 8, Czech Republic, ${ }^{4}$ Material Physics Laboratory, LUT University, Yliopistonkatu 34, 53850 Lappeenranta, Finland, ${ }^{5}$ Faculty of Mathematics and Physics, Charles University, Ke Karlovu 5, 12116 Prague 2, Czech Republic, ${ }^{6}$ Institut Laue-Langevin, 71 avenue des Martyrs, CS 20156, 38042 Grenoble cedex 9, France.

vertat@fzu.cz

Ni-Mn-Ga-based Heusler alloys are broadly studied for their magnetic shape memory (MSM) functionality originating from coupling between ferroelastic and ferromagnetic orders. The ferroelastic order is established after martensitic transformation. Formed ferroelastic domains with different orientation are separated by twin boundaries. In modulated phases, these boundaries are extremely mobile and can be manipulated by magnetic field. Thanks to these, the single crystals of five-layered modulated $10 \mathrm{M}$ martensite of Ni-Mn-Ga exhibits magnetically induced reorientation (MIR) of ferroelastic (twin) domains in a moderate field of the order of $0.1 \mathrm{~T}$ $[1,2]$. This results in $6 \%$ magnetic field induced strain (MFIS) down to liquid helium temperature [3]. Such unique behaviour makes the $10 \mathrm{M}$ martensite a perfect candidate for applications in actuators, sensors and energy harvesters.

The ferroelastic microstructure represents a challenge for proper determination of martensite phase structure. Due to the modulated nature together with complex hierarchical twinning (compound and type $I$ and II a/c twins; and non-conventional twins) [4, 5], the structure of the $10 \mathrm{M}$ martensite has not yet been completely solved. There is even an ongoing discussion about the nature of the modulation where two main concepts are considered: i) general crystallographic wave modulation approach, and ii) nanotwinning. As the structural modulation seems to be the critical factor for the extremely high twin boundary mobility [5], the problem is pressing.

Using the X-ray and neutron diffraction, we investigated on the character and temperature evolution of $10 \mathrm{M}$ martensite phase. We found transition from commensurate to incommensurate $10 \mathrm{M}$ modulated structure in $\mathrm{Ni}_{50} \mathrm{Mn}_{27} \mathrm{Ga}_{22} \mathrm{Fe}_{1}$ single crystal [6]. The modulation vector gradually increases upon cooling from commensurate $\mathbf{q}=(2 / 5) \mathbf{g}_{110}$, where $\mathbf{g}_{110}$ is the reciprocal lattice vector, to incommensurate with $\mathbf{q}$ up to pseudo-commensurate $\mathbf{q}=(3 / 7) \mathbf{g}_{110}$. Further cooling results in transition to $14 \mathrm{M}$ with $\mathbf{q}=2 / 7 \mathbf{g} 110$. Upon heating, reverse changes of the commensurate-incommensurate transition are observed with a thermal hysteresis of $\approx 60 \mathrm{~K}$. We detected the same hysteretic behaviour in the electrical resistivity and the effective elastic modulus. Scanning electron microscopy showed that the changes are accompanied by the refinement of the $a / b$ laminate.

Furthermore, we observed continuous modulation changes within the $10 \mathrm{M}$ martensite of wide range of $\mathrm{Ni}-\mathrm{Mn}-\mathrm{Ga}(-\mathrm{Fe})$ compositions that undergo the Austenite $\rightarrow 10 \mathrm{M} \rightarrow 14 \mathrm{M}$ martensite transition sequence. Based on these observations, we suggest that the commensurate state is a metastable form of $10 \mathrm{M}$ martensite. Upon cooling, this phase evolves through nanotwinning into a more irregular and more stable incommensurate structure, further supported by recent high-resolution TEM observation [7].

[1] Ullakko, K., Huang, J. K., Kantner, C. \& Handley, R. C. O. (1996) Appl. Phys. Lett. 69, 1966-8.

[2] Kellis, D., Smith, A., Ullakko, K. \& Müllner, P. (2012) J. Cryst. Growth 359, 64-68.

[3] Heczko, O., Kopecký, V., Sozinov, A. \& Straka, L. (2014) Appl. Phys. Lett. 103, 198-211.

[4] Straka, L., et al. (2011) Acta Mater. 59, 7450-63.

[5] Seiner, H., Straka, L. \& Heczko, O. (2013) J. Mech. Phys. Solids 64, 072405.

[6] Veřtát, P., et al. (2021) J. Phys.: Condens. Matter 33, 265404.

[7] Ge, Y. et al., "Transitions between austenite and martensite structures in Ni50Mn25Ga20Fe5 thin foil", available at: http://dx.doi.org/10.2139/ssrn.3813433

\section{Keywords: magnetic shape memory; phase transformations; Ni-Mn-Ga; twinned microstructure; structural modulation}

This work was supported by Operational Programme Research, Development and Education financed by the European Structural and Investment Funds and the Czech Ministry of Education, Youth and Sports, project number SOLID21 CZ.02.1.01/0.0/0.0/16_019/0000760. P.V. thanks for the support by the Grant Agency of the Czech Technical University in Prague, grant number SGS19/190/OHK4/3T/14. We acknowledge the Institut Laue-Langevin and the project LTT20014 financed by the Ministry of Education, Youth and Sports, Czech Republic, for the provision of neutron radiation facilities.

Acta Cryst. (2021), A77, C399 